\title{
approche expérimentale du fluage en compression triaxiale de deux argiles intactes
}

\author{
par \\ Félix B., \\ Ingénieur \\ Kenana A., \\ Élève chercheur \\ Laboratoire central des Ponts et Chaussées, Paris \\ Section des Fondations
}

\section{Introduction}

L'essai triaxial de fluage n'est pas un essai usuel en mécanique des sols. Pour des raisons de durée d'essai et de codification du mode opératoire l'essai à vitesse de déformation imposée lui est préféré. L'étude du cisaillement des argiles sous charge constante ou imposée (essai de fluage) est pourtant fondamentale. Dans la nature, au sein d'une pente instable ou sous un ouvrage ce sont généralement les charges qui sont imposées et non les vitesses de déformation ou même les déformations elles-mêmes.

Comme tous les matériaux réels, les sols ont des lois de comportement complexes. La nature discontinue des arrangement de particules qui constituent son squelette n'est généralement pas prise en compte dans les modèles de comportement. Des contraintes effectives sont définies par soustraction de la pression de l'eau aux composantes normales des contraintes. Ces contraintes dites aussi intergranulaires sont utilisées dans les formulations de cet article, le squelette étant supposé être un milieu homogène continu.

Les modèles rhéologiques rencontrés dans la littérature sont associés à un type d'essai bien déterminé. II existe des modèles unidimensionnels adaptés aux essais codométriques ou de cisaillement direct, c'est le cas des modèles de S. R. Meschyan issus de la théorie du fluage héréditaire (visco-élasticité). Les lois de comportement tenant compte des effets du temps, dérivées des modèles cam clay, sont bien adaptées aux conditions de l'essai triaxial qui permet d'imposer des chargements axisymétriques dont l'axe se confond avec celui de l'éprouvette cylindrique. Ces modèles soní brièvemeni rappelés dans cet aiticie.

Les deux argiles intactes utilisées pour les essais ont des caractéristiques très différentes. Elles sont soumises à des chargements qui excèdent largement les contraintes appliquées in situ, leur comportement est par conséquent caractéristique de leur domaine normalement consolidé.
Le programme d'essais de fluage a été conçu pour mettre en évidence les caractéristiques suivantes du fluage de l'argile :

- Influence de l'anisotropie de structure et de la dilatance de ce matériau.

- Écoulement et rupture sous charge constante.

- Domaine d'application d'un modèle visco-élastique.

2 Présentation d'un modèle visco-élastique et d'un modèle visco-plastique adapté au comportement des argiles

\subsection{La théorie du fluage héréditaire}

Pour l'expérimentateur, la théorie du fluage héréditaire présente un grand intérêt. Elle englobe en tant que cas particuliers les modèles visco-élastiques linéaires basés sur la combinaison d'éléments rhéologiques ressorts et amortisseurs - sans en avoir les inconvénients relatifs à la détermination des paramètres. Dans le cas d'une sollicitation unidimensionnelle, un modèle non-linéaire est caractérisé par deux fonctions $C(t)$ et $F(\sigma)$ dont la signification physique est évidente, Félix (1980).

L'équation de comportement s'écrit, pour un essai de fluage :

$$
\varepsilon(\mathrm{t})=\int_{0}^{t} \frac{\partial c(\mathrm{t}-\tau)}{\partial \mathrm{t}} \mathrm{F}[\sigma(\tau)] \mathrm{d} \tau .
$$

Le matériau ne présentant pas de déformation strictement instantanée, $\mathrm{C}(\mathrm{t})$ décrit la déformation sous une charge constante $\sigma_{1}$ tel que $F\left(\sigma_{1}\right)=1$ et $F(\sigma)$ est la fonction de contrainte caractéristique de la nonlinéarité du matériau. Ce modèle est intéressant lorsque la condition: vitesse de déformation initiale infinie est satisfaite.

Les équations du fluage se généralisent facilement au cas général à trois dimensions. 
2.2 Schéma de comportement élasto-viscoplastique dérivé du modèle Cam clay

Les paramètres classiques du modèle cam clay sont les variables de contraintes $p^{\prime}=\frac{\sigma_{1}^{\prime}+2 \sigma_{3}^{\prime}}{3}$ et $q=\sigma_{1}^{\prime}-\sigma_{3}^{\prime}$ et l'indice des vides e du sol, $\sigma_{1}^{\prime}$ et $\sigma_{3}^{\prime}$ étant les contraintes effectives principales correspondant respectivement aux directions verticales et radiales des éprouvettes. Le modèle original dû à Roscoe, Schofield et Wroth (1958) est élasto-plastique avec écrouissage.

II propose un schéma unique de comportement des argiles remaniées valable pour l'interprétation des essais de laboratoire classiques en mécanique des sols et de l'essai triaxial en particulier.

Les concepts de base sont:

- l'état critique : c'est un état caractérisé par un cisaillement continu sans variation de volume sous contrainte constante $p^{\prime}, q$;

- la limite d'écoulement plastique : c'est la surface dans l'espace p', q, e à l'intérieur de laquelle le comportement du sol est toujours élastique. Si le point représentatif de l'état de l'échantillon est sur la limite d'écoulement plastique, à tout accroissement de contrainte au-delà de la limite correspond un accroissement de déformation entièrement plastique et un écrouissage fonction de la déformation volumique plastique (de la variation de l'indice des vides correspondante);

Les courbes représentatives des états critique et limite sont représentées sur la figure 1 , qui correspond à un modèle adapté du précédent pour prendre en compte les effets du temps.

\subsubsection{Modèle pour argiles remaniées isotropes}

Le modèle schématisé sur la figure 1 a été proposé par l'université de Kyoto: Adachi et Okano (1974), Akai, Adachi et Fujimoto (1977). II constitue une extension aux matériaux élasto-visco-plastiques du modèle de Cam-clay par introduction de nouveaux concepts dus à Perzyna (1963).

L'équation de la courbe d'état limite statique est de la forme :

$$
F_{s}\left(\sigma_{i j}^{\prime}, \varepsilon_{i, j}^{P_{j}}\right)=k_{\mathrm{s}}
$$

et celle de la courbe d'état limite dynamique :

$$
F_{d}\left(\sigma_{i j}^{\prime}, \varepsilon_{i j}^{P}, \dot{\varepsilon}_{i j}\right)=k_{d}
$$

$\mathrm{k}_{\mathrm{s}}$ est le paramètre d'écrouissage statique qui dépend uniquement des déformations volumiques plastiques $\varepsilon_{V}^{P}$,

$k_{\mathrm{d}}$ est un paramètre d'écrouissage dynamique. II dépend des vitesses de déformations et des déformations $\varepsilon_{V}^{P}$.

La vitesse d'écoulement visco-plastique est associée au potentiel plastique dynamique défini par (2) suivant l'équation d'écoulement (4) dans laquelle intervient la fonction excès de contrainte $F=\frac{F_{d}-F_{s}}{F_{a}}$.

$$
\dot{\varepsilon}_{i j}=\frac{1}{\eta}<\phi(F)>\frac{\partial F_{2}}{\partial \sigma_{i j}^{\prime}} \text { pour } F>0 .
$$

$\eta$ est la viscosité dépendant de la température.

$$
\begin{array}{rrr}
<\phi(F)>=0 & \text { si } & F<0 \\
\phi(F) & \text { si } & F>0 .
\end{array}
$$

La courbe d'état critique est une droite associée au critère de rupture de Mohr-Coulomb.

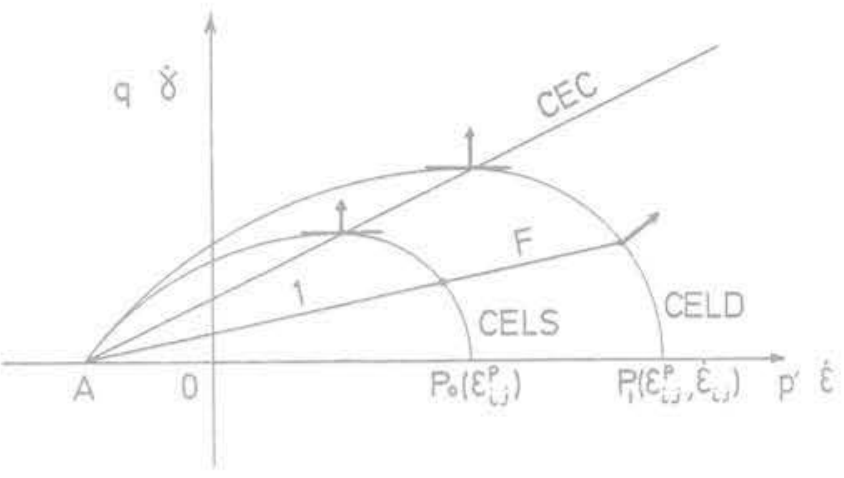

Fig. 1 Courbes d'état critique CEC - Courbes d'état limite statique CELS et dynamique CELD

du type de celles proposées dans Adachi et al, 74.77

- Réponse du modèle dans le cas d'un essal triaxial de fluage drainé.

Au cours d'un tel essai les contraintes effectives p' et q restent constantes (le chargement est constant, les pressions interstitielles sont dissipées par drainage).

- Si le point de coordonnées $p^{\prime}, q$ est situé à l'intérieur de la courbe d'état limite statique (C. E. L.S.) les déformations sont élastiques instantanées (non visqueuses).

- Si le point p', q est situé à l'extérieur de cette courbe, deux cas se présentent:

$f^{\text {er }}$ cas: le point $p^{\prime}$, q est en dessous de la courbe d'état critique. Dans ce cas, des déformations visco-plastiques se développent avec des vitesses d'autant plus grandes que la courbe d'état limite dynamique (C. E. L.D.) est plus largement extérieure à la C.E.L.S. Il est généralement supposé dans les formulations mathématiques du modèle que les 2 courbes C. E. L.S. et C.E.L.D. se déduisent l'une de I'autre par homothétie de centre A.

Le développement des déformations volumiques plastiques entraine par écrouissage l'élargissement de la C.E. L.S, qui tend vers la C.E.L.D. Les vitesses de déformation diminuent corrélativement et tendent vers zéro lorsque les deux courbes se confondent et passent par le point $p^{\prime}, q$ caractéristiques du chargement.

$2^{\circ}$ cas : le point p', q est au-dessus de la courbe d'état critique, le matériau se radoucit, c'est-à-dire que la C.E.L.S. diminue en diamètre, l'échantillon ne peut atteindre un état d'équilibre, c'est la rupture.

\subsubsection{Modèles pour argiles intactes anisotropes}

Les concepts fondamentaux du modèle Cam clay initial ont été modifiés pour simuler le comportement des argiles non remaniées par Tavenas et Leroueil (1977). Ils ont formulé un modèle Ylight pour les argiles sensibles et cimentées du Québoc, qui s'est avéré caractéristique du comportement d'autres argiles, Shahanguian (1981).

Ce modèle tient compte de :

- l'anisotropie de comportement de l'argile intacte,

- la préconsolidation du matériau en place sur ses caractéristiques visco-plastiques. 
Les modèles de comportement visqueux pour les sols sont très nombreux. Les concepts de superposition utilisés dans la théorie du fluage héréditaire ont été généralisés : théorie endochronique (Valanis, (1971), Ansal, Bazant et Krizek (1979)) qui aboutit à des modèles visco-plastiques sans seuil de plasticité. De nombreuses équations de comportement ont été formulées sur la base des concepts du modèle Cam clay, qui tiennent compte de la dilatance des argiles (de la diminution de volume induite par une augmentation des contraintes de cisaillement), de l'anisotropie induite par la reconsolidation, etc., Sekiguchi, Ohta (1977), Enfin, beaucoup d'autres modèles viscoplastiques sortent du cadre de cette étude et ne peuvent étre examinés ici.

Les notions rapidement présentées dans cet article vont être confrontées aux résultats expérimentaux obtenus pour deux argiles non remaniées.

\section{Dispositif expérimental et mode opératoire des essais de fluage triaxiaux}

\subsection{Les problèmes expérimentaux, la mesure des déformations radiales}

Les essais de fluage triaxial drainé ont été effectués à l'aide de cellule de construction classique. Le fluide de confinement est de l'eau désaérée et les charges verticales sont appliquées - soit en montant les cellules triaxiales sur des bâtis cedométriques (la force verticale est appliquée sur l'éprouvette par l'intermédiaire d'un bras de levier au moyen d'un jeu de poids) - soit en plaçant un étrier sur le piston de la cellule et en le chargeant directement à l'aide de poids.

La déformation verticale des éprouvettes de sol est mesurée directement par un comparateur solidaire du piston. La pression interstitielle est mesurée à la base des éprouvettes tandis que le drainage est assuré par la surface supérieure reliée à un circuit ouvert à la pression atmosphérique (pas de contrepression), comportant une burette (essais sur l'argile des Flandres) ou un volumètre (essais sur l'argile de Cubzac-les-Ponts).

Le volume drainé ne peut être considéré pour le calcul de la déformation volumique des échantillons: les essais de fluage étant de longue durée (150 jours pour certains essais sur l'argile des Flandres), la quantité d'eau qui traverse la membrane de $1 \mathrm{~mm}$ d'épaisseur ne peut être considérée comme négligeable.

Trois bagues équipées de vernier telles que celle représentée sur la figure 2 ont été montées autour de chaque échantillon, de façon à mesurer avec une précision de l'ordre de $\pm 0,03 \mathrm{~mm}$ la variation du diamètre au premier quart de la hauteur de l'éprouvette, à mi-hauteur et au quart supérieur.

Les déformations radiales ainsi mesurées sont utilisées pour calculer les déformations volumiques et déviatoriques et pour effectuer des corrections de poids appliqués au piston des cellules de façon à maintenir les contraintes constantes malgré les variations de la section des éprouvettes Félix (1981).

\subsection{Mode opératoire des essais de fluage sur eux argiles de caractéristiques très diffé-} rentes

L'argile des Flandres prélevée par vérinage entre 4 et $6 \mathrm{~m}$ de profondeur à Winnezeele (Nord de la France)

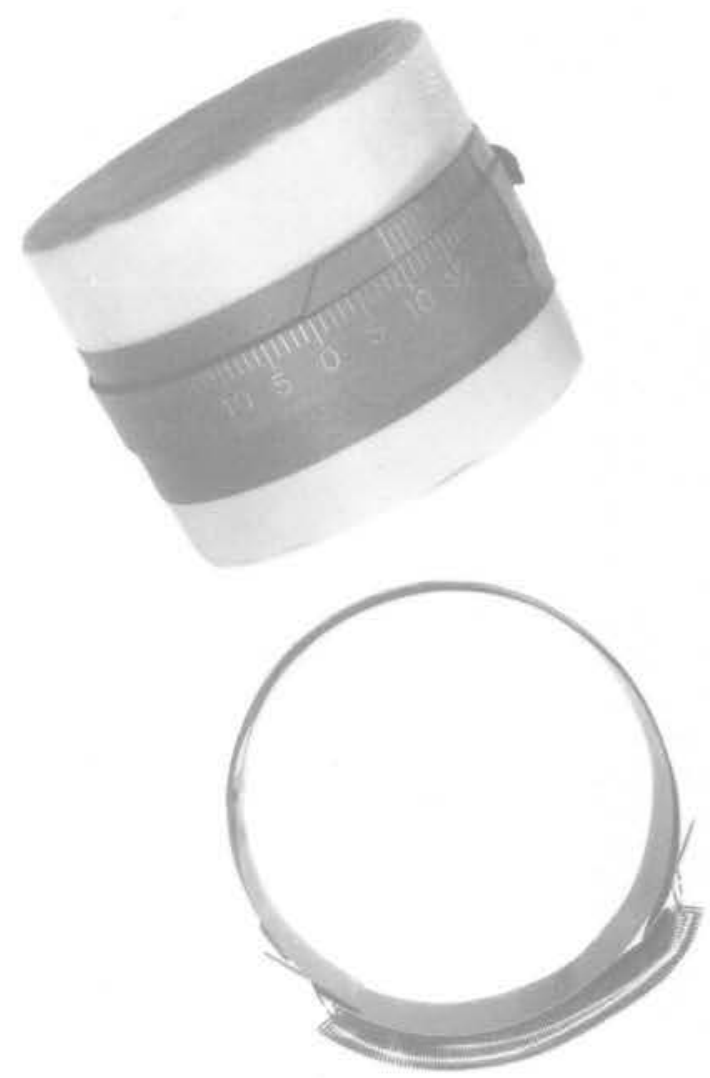

Fig. 2 Bague de mesure des déformations radiales des éprouvettes

est une argile raide, bleue-grise, fortement surconsolidée.

L'argile molle a été prélevée au carottier à piston stationnaire à Cubzac-les-ponts (Gironde, Sud-Ouest de la France) entre 4 et $5 \mathrm{~m}$ de profondeur, cette argile légèrement organique est faiblement surconsolidée.

Les caractéristiques des deux argiles sont comparées sur le tableau 1:

Tableau 1

\begin{tabular}{lccc}
\hline \multicolumn{1}{c}{ Caractéristiques } & Symb. & $\begin{array}{c}\text { A. des } \\
\text { Flandres }\end{array}$ & $\begin{array}{c}\text { A. de } \\
\text { Cubzac }\end{array}$ \\
\hline $\begin{array}{l}\text { Poids } \\
\text { volumique }\end{array}$ & $\gamma$ & $19 \mathrm{kN} / \mathrm{m}^{3}$ & $15 \mathrm{kN} / \mathrm{m}^{3}$ \\
\hline $\begin{array}{l}\text { Poids } \\
\text { volumique sec }\end{array}$ & $\gamma_{\mathrm{d}}$ & $14,3 \mathrm{kN} / \mathrm{m}^{3}$ & $8,6 \mathrm{kN} / \mathrm{m}^{3}$ \\
\hline $\begin{array}{l}\text { Teneur } \\
\text { en eau }\end{array}$ & W & $35 \%$ & $74 \%$ \\
\hline $\begin{array}{l}\text { Indice de } \\
\text { plasticité }\end{array}$ & $\mathrm{I}_{\mathrm{p}}$ & $33 \mathrm{à} 66$ & 60 \\
\hline $\begin{array}{l}\text { Cohésion } \\
\text { effective }\end{array}$ & $\mathrm{c}^{\prime}$ & $5 \mathrm{kPa}$ & $14 \mathrm{kPa}$ \\
\hline $\begin{array}{l}\text { Angle de' frott. } \\
\text { effectif }\end{array}$ & $\varphi^{\prime}$ & $27^{\circ}$ & $23^{\circ}$ \\
\hline $\begin{array}{l}\text { Pression de } \\
\text { préconsolidation }\end{array}$ & $\sigma_{\mathrm{p}}^{\prime}$ & $150 \mathrm{kPa}$ & $38 \mathrm{kPa}$ \\
\hline
\end{tabular}


3.2.1 Les essais sur l'argile des Flandres ont été eif́ctués sur des éprouveties de $75 \mathrm{~mm}$ de diamètre et $150 \mathrm{~mm}$ de hauteur. Tous iss échantilions ont été préalablement consolidés sous une pression hydrostatique supérieure à $\sigma_{p}^{\prime}$ pendant 10 jours.

Les essais de fluage au cisaillement ont été réalisés à $p^{\prime}=\frac{\sigma_{3}^{\prime}+2 \sigma_{3}^{\prime}}{3}$ constant et égal à la pression hydrostatique de consolidation.

Deux types d'essais ont été réalisés :

a) 4 échantillons après consolidation sous $\mathrm{p}^{\prime}=500 \mathrm{kPa}$ ont été soumis instantanément à une contrainte déviatorique $\mathrm{q}=\sigma_{1}^{\prime}-\sigma_{3}^{\prime}$ égale à $315 \mathrm{kPa}$, $270 \mathrm{kPa}, 225 \mathrm{kPa}$ et $180 \mathrm{kPa}$ représentant respectivement $70 \%, 60 \%, 50 \%$ et $40 \%$ du déviateur de rupture présumé calculée à partir des caractéristiques $c^{\prime}$ et $\varphi^{\prime}$ de l'argile, soit q $q_{\text {rupt }}=450 \mathrm{kPa}$.

Les déformations ainsi que les pressions interstitielles et le volume drainé ont été mesurés pendant 170 jours.

b) 3 échantillons ont été consolidés sous 200,350 et $500 \mathrm{kPa}$ respectivement puis chargés par paliers de contrainte déviatorique, appliqués instantanément à intervalle de 7 jours et égaux à $10 \%$ du déviateur de rupture présumé, soit $180 \mathrm{kPa}, 315 \mathrm{kPa}$ et $450 \mathrm{kPa}$ respectivement. Le chargement est ainsi mené jusqu'à la rupture. Les états de contrainte successifs appliqués à ces trois échantillons sont représentés par des points sur les diagrammes $p^{\prime}$, q des figures 5 et 6 .

\subsubsection{Les essais sur l'argile molle de Cubzac-les- ponts}

Ces essais complètent les précédents, qui avaient mis en évidence l'importance de l'anisotropie et de la dilatance des argiles intactes. Deux types de chargement par paliers égaux de contraintes isotropes $\Delta p^{\prime}$ et déviatorique $\Delta q$ ont été réalisés de façon à imposer des chemins de déformations proches des chemins suivants :

a) déformations déviatoriques sans déformation volumique,

b) déformations volumiques sans déformation déviatorique.

Tous les échantilions de $55 \mathrm{~mm}$ de diamètre et de $110 \mathrm{~mm}$ de hauteur ont été préalablement consolidés sous une contrainte hydrostatique de $80 \mathrm{kPa}$ pendant 36 jours.

a) Pour les essais de fluage induisant essentiellement des déformations de cisaillerrent, les rapports suivants de variation de $\Delta p^{\prime}$ et de $\Delta q$ ont été utilisés pour 3 échantillons différents :

$\Delta p^{\prime}=\chi \Delta q \quad \chi=-0,36 ; \quad-0,254 ; \quad-0,167$, la composante isotrope decroissant plus ou moins rapidement à chaque palier. Quatre paliers successifs ont été appliqués à intervalle de 14 jours de façon à atteindre l'état critique de rupture au $4^{\circ}$ palier.

b) Pour les essais de fluage induisant essentiellement des déformations volumiques, les rapports suivants de $\Delta \mathrm{p}^{\prime}$ et de $\Delta \mathrm{q}$ ont été utilisés pour 3 échantilions nifférents .

$$
\Delta \mathrm{q}=\zeta \Delta \mathrm{p}^{\prime} \quad \zeta=0,75 ; \quad 0,30 ; \quad 0 .
$$

Après consolidation, quatre paliers égaux d'une durée de 14 jours ont été appliqués, avec pour chacun $\Delta \sigma_{1}^{\prime}=40 \mathrm{kPa}$.

Les composantes $p^{\prime}, q$ des contraintes correspondant aux différents paliers de chargements des 6 échantil-
Ions des séries a et b sont représentés par des points sur les figures 8 et 9 .

\section{Analyse visco-élastique du fluage au cisail- lement de l'argile des Flandres}

\subsection{Superposition des déformations, non- linéarité de la relation contrainte- déformation}

A partir de $\varepsilon_{1}$ et $\varepsilon_{3}$ les déformations verticales et radiales sont définies:

les déformations volumiques : $\theta=\varepsilon_{1}+2 \varepsilon_{3}$

et les déformations déviatoriques : $D=\varepsilon_{1}-\varepsilon_{3}$.

Suivant les conventions d'usage en mécanique des sols $\varepsilon_{1}, \varepsilon_{2}$ et $\theta$ sont positives en compression.

La figure 3a représente en fonction du temps les déformations déviatoriques des échantillons soumis à des contraintes déviatoriques constantes égales à $40 \%, 50 \%, 60 \%$ et $70 \%$ du déviateur de rupture $Q_{\text {rupt }}$ ainsi que celles d'un échantillon soumis par paliers d'une durée de 7 jours à un déviateur croissant par incréments égaux à $10 \%$ de $q_{\text {rupr. }}$

Sur la figure $3 \mathrm{~b}$, la déformation déviatorique sous charge constante après 7 jours et celle enregistrée à la fin de chaque palier (carrés noirs) sont tracées en fonction des contraintes déviatoriques.

Ces résultats amènent les remarques suivantes :

- Les vitesses de fluage au cisaillement, quasi infinies au moment du chargement, décroissent ensuite vers des valeurs nulles.

- Le fluage est de type amorti même pour les niveaux de contraintes élevées proches des conditions de rupture $\left(70 \%\right.$ de $\left.\mathrm{q}_{\text {rupt }}\right)$ définies à partir des paramètres de cisaillement drainé classique $c^{\prime}$ et $\varphi^{\prime}$. Ce résultat est conforme à ceux de Bishop Lovenbury (69), Shibata Karube (69), Schultze (71), Robinet (78).

Pour l'essai de fluage sous chargement par paliers on observe des déformations particulièrement importantes sous le premier palier, qui sont induites par la mise en contact brutale sous les premières charges verticales de l'argile dont la surface est parfaitement plane et des embases rugueuses couvertes de papier filtre. Ce phénomène se produit vraisemblablement pour tous les essais, et introduit une erreur qui aurait été évitée si les échantiilons avaient été consolidés anisotropiquement et à ce titre soumis à un chargement axial prealable.

La relation contrainte-déformation pour une valeur donnée du temps ( 7 jours) est non linéaire comme le montre la figure $3 \mathrm{~b}$. Elle présente un seuil égal à $30 \%$ environ du déviateur de rupture sous lequel les déformations sont très faibles. 11 y a entre les relations obtenues par les 4 chargements constants et par chargement par paliers une approximative concordance.

II a été montré, Félix (80) que dans ce cas les déformations de fluage sous un chargement monotone croissant, se prêtaient à une interprétation à l'aide d'un modèle basé sur le principe de superposition, c'est-à-dire un modèle visco-élastique et ceci bien que les déformations de cisaillement de l'argile des Flandres soient, dans le cas étudié, essentiellement visco-plastiques.

Si $D(t)$ est la fonction de fluage qui décrit la déformation déviatorique sous la charge $\mathrm{q}_{1}$, et $\mathrm{F}(\mathrm{q})$ la 

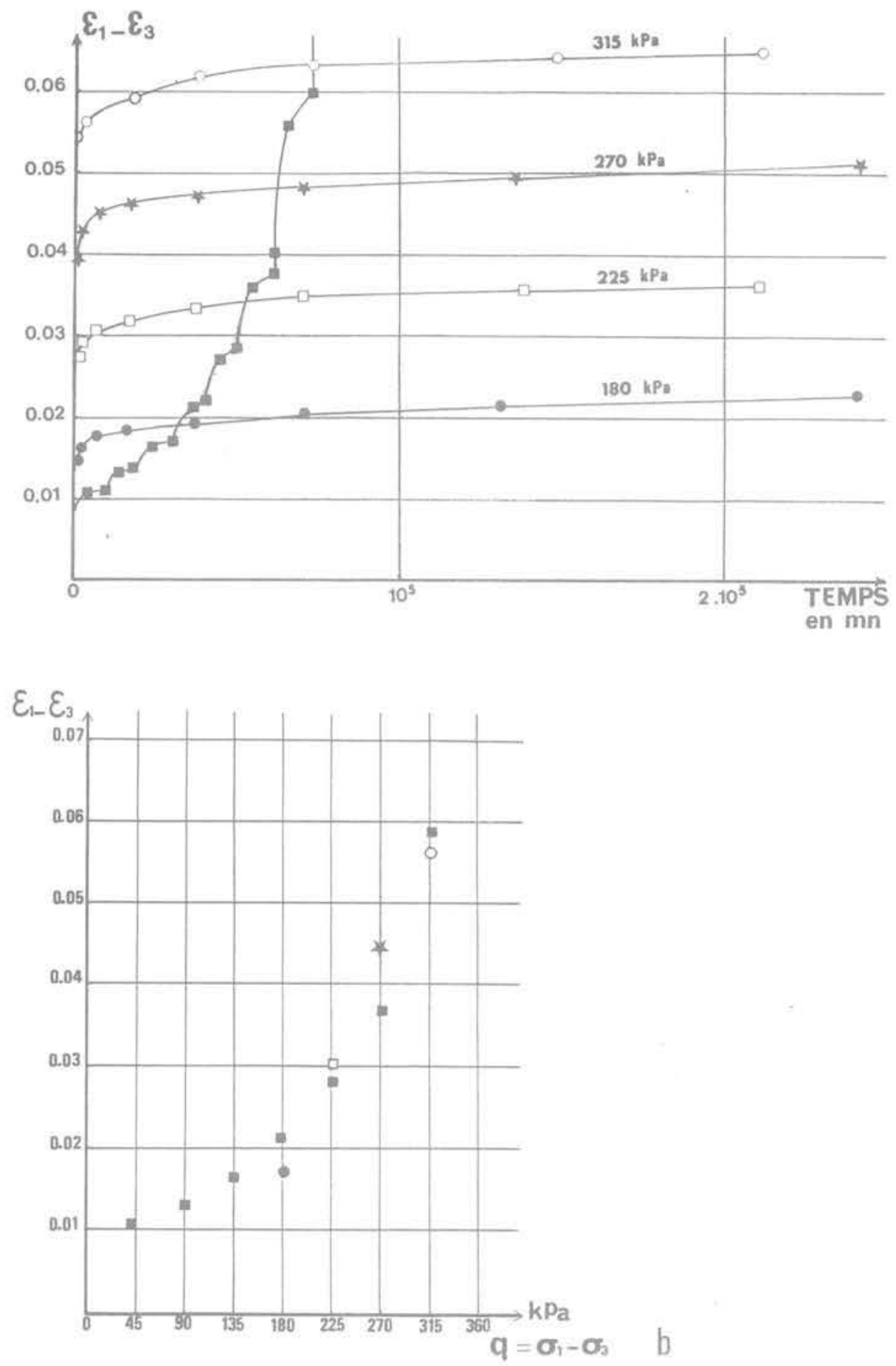

Fig. 3 Déformation de cisaillement fonction du temps (a) et fonction de la contrainte déviatorique (b) pour quatre charge. ments constants sous $40 \%, 50 \%, 60 \%$ et $70 \%$ du déviateur de rupture et pour un chargement par paliers égaux à $10 \%$ de celui-ci

fonction de contrainte rapport d'affinité entre les courbes sous les charges $q$ et $q_{1}:\left(q<q_{\text {rupt }}\right)$

$$
F(q)=\frac{D_{q}\left(t_{0}\right)}{D\left(t_{0}\right)} \text { avec } F\left(q_{1}\right)=1 \text { et } F(0)=0
$$

$t_{0}$ étant une valeur arbitraire du temps de fluage. Sous charge constante q la déformation déviatorique est simplement décrite par :

$$
D_{q}(t)=D(t) \cdot F(q)
$$

Sous un chargement par paliers où q prend successivement au temps $t_{i}$ les valeurs $q_{i}$, la fonction de contrainte prend au temps $t$ la valeur:

$$
F(q)=\sum_{i=1}^{n}\left[F\left(q_{i}\right)-F\left(q_{i-1}\right)\right] h\left(t-t_{i}\right)
$$

avec $h(t)$ la fonction échelon de Heaviside

$$
\begin{aligned}
& h(t)=1 \text { pour } t>0 \\
& h(t)=0 \text { pour } t<0 .
\end{aligned}
$$

Compte tenu de l'équation (1):

$$
\begin{aligned}
D_{q n}(t)=\int_{0}^{t} \frac{\partial D(t-\tau)}{\partial \tau} & F(q) d \tau \\
& =\sum_{i=1}^{n} D\left(t-t_{i}\right)\left[F(q)-F\left(q_{i}-1\right)\right]
\end{aligned}
$$

qui décrit le fluage déviatorique sous un chargement de cisaillement croissant appliqué après consolidation isotrope sous la même pression $\mathrm{p}^{\prime}$. 


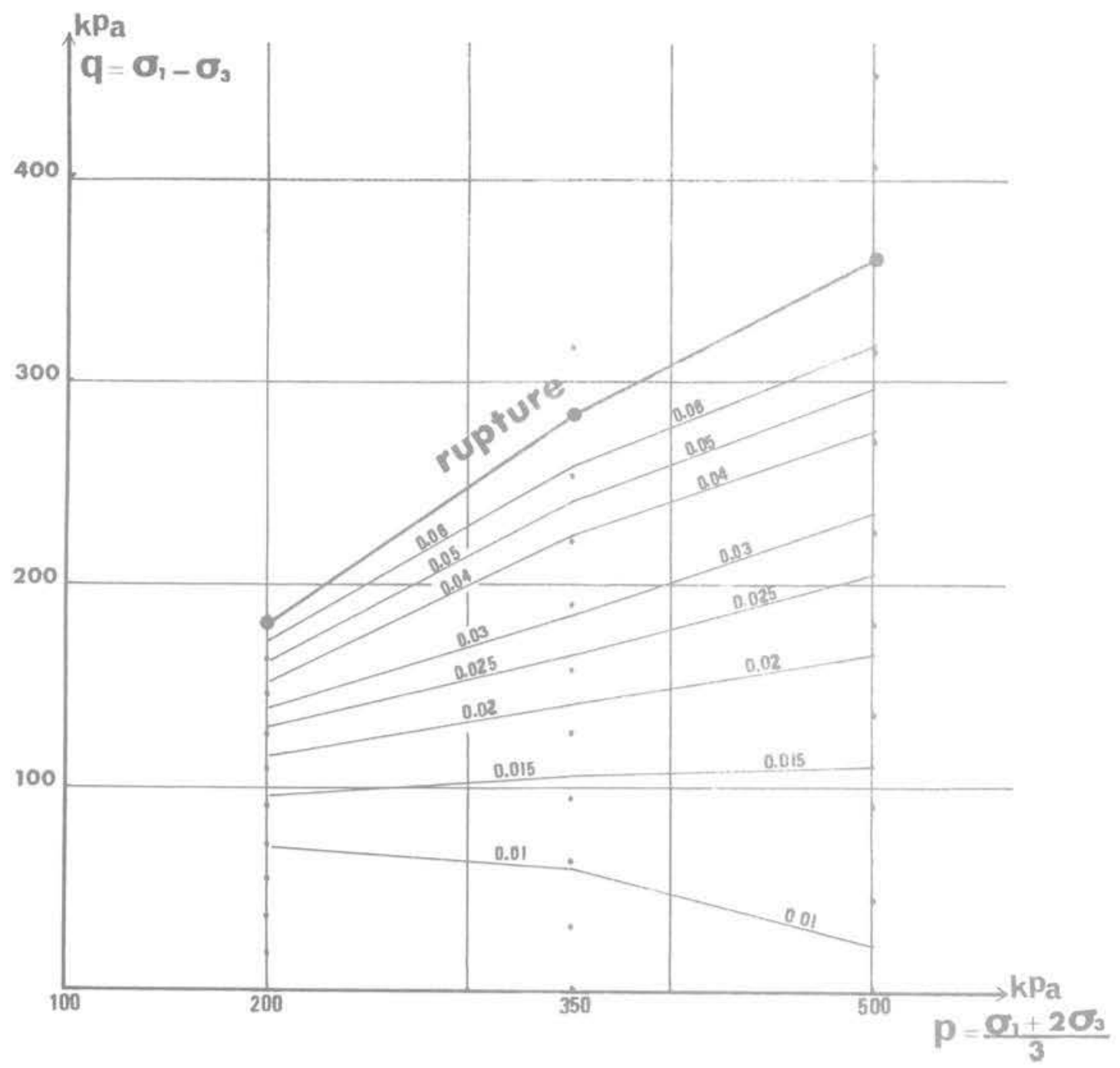

Fig. 4 Points représentatifs des chargements p', $q$ appliqués au cours des trois essais de fluage au cisaillement par paliers et courbes d'égales déformations déviatoriques

4.2 Formulation visco-élastique du fluage au cisaillement sous différentes composantes isotropes du chargement maintenues constantes

Sur la figure 4 sont représentés par des points dans le diagramme $p^{\prime}$, q les états de contraintes successivement appliqués au cours des 3 essais de fluage sous chargement de cisaillement par paliers après reconsolidation sous trois contraintes hydrostatiques différentes : $200 \mathrm{kPa}, 350 \mathrm{kPa}$ et $500 \mathrm{kPa}$.

Par interpolation entre les déformations déviatoriques D obtenues en fin de paliers, les courbes joignant les états de contraintes correspondant aux mêmes valeurs de $\mathrm{D}$ ont été tracées. On observe que :

- Le déviateur de rupture représente les fractions suivantes du déviateur calculé à partir de $c^{\prime}$ et $\varphi^{\prime}$ :

$$
\begin{array}{rl}
\mathrm{p}^{\prime}=200 \mathrm{kPa} & 100 \% \text { du déviateur présumé } \\
\mathrm{p}^{\prime}=350 \mathrm{kPa} & 90 \% \\
\text { et } \mathrm{p}^{\prime}=500 \mathrm{kPa} & 80 \% .
\end{array}
$$

Les points $p^{\prime}, q_{\text {rupt }}$ se trouvent approximativement sur une droite d'équation :

$$
q=M\left(p^{\prime}+a\right)
$$

dont la pente $M=\frac{6 \sin \varphi^{\prime}}{3-\sin \varphi^{\prime}}$ correspond à un angle de frottement interne $\varphi^{\prime}$ plus petit que celui déterminé par des essais triaxiaux drainés classiques.
- Quel que soit l'état de contrainte qui y mène, la rupture se produit pour une déformation déviatorique identique de l'ordre de $D=0,08$. Ce résultat recoupe ceux de Vyalov (57), Goldstein (57) et Murayama Shibata (61).

- Les courbes p', q correspondant aux mêmes déformations déviatoriques forment approximativement un faisceau de droites concourantes au point d'abscisse à l'origine de la droite de rupture d'équation (9). En d'autres termes la déformation en fin de palier de fluage au cisaillement, de 7 jours de durée, s'exprime en fonction du rapport $\frac{q}{q_{\text {rupt }}}$.

- La formulation de l'équation (1) du fluage héréditaire peut, en s'appuyant sur les résultats précédents et sur ceux de Meschyan (76) être généralisée aux déformations déviatoriques sous une contrainte de consolidation préalable $\mathrm{p}^{\prime}$ quelconque.

La fonction de contrainte se transforme comme suit :

$$
F(q)=\phi\left[\frac{q}{M(p+a)}\right]=\phi\left[\frac{q}{q_{\text {rupt }}}\right] .
$$

\subsection{Dilatance de l'argile des Flandres}

La dilatance de l'argile des Flandres, c'est-à-dire les déformations volumiques provoquées par les contraintes de cisaillement, est mise en évidence sur la figure 5 . Les déformations volumiques y sont tracées en 


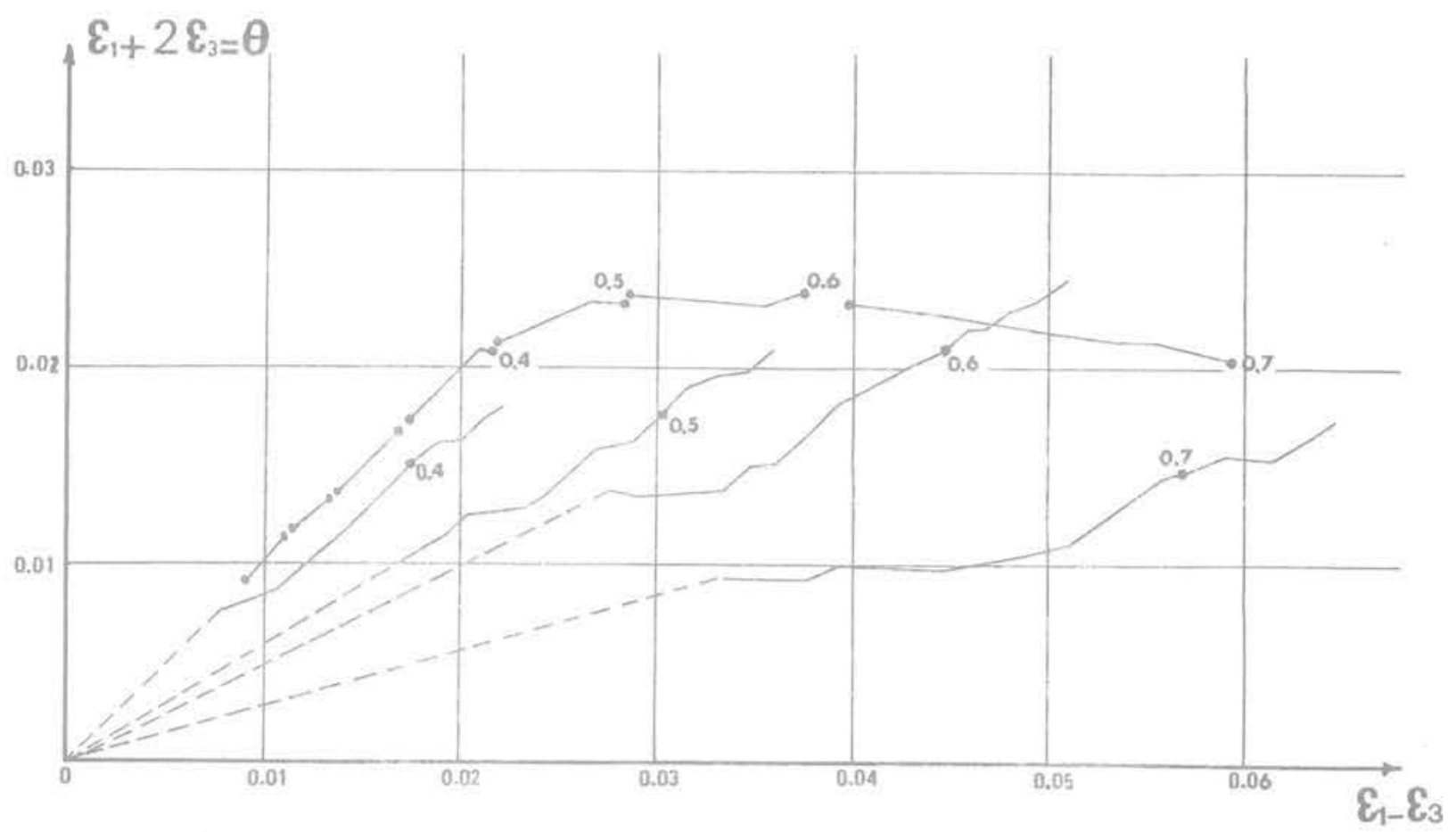

Fig. 5 Evolution de la déformation volumique en fonction de la déformation déviatorique qui se développe au cours de cinq essais de fluage au cisaillement sous charges constantes ou variant par paliers

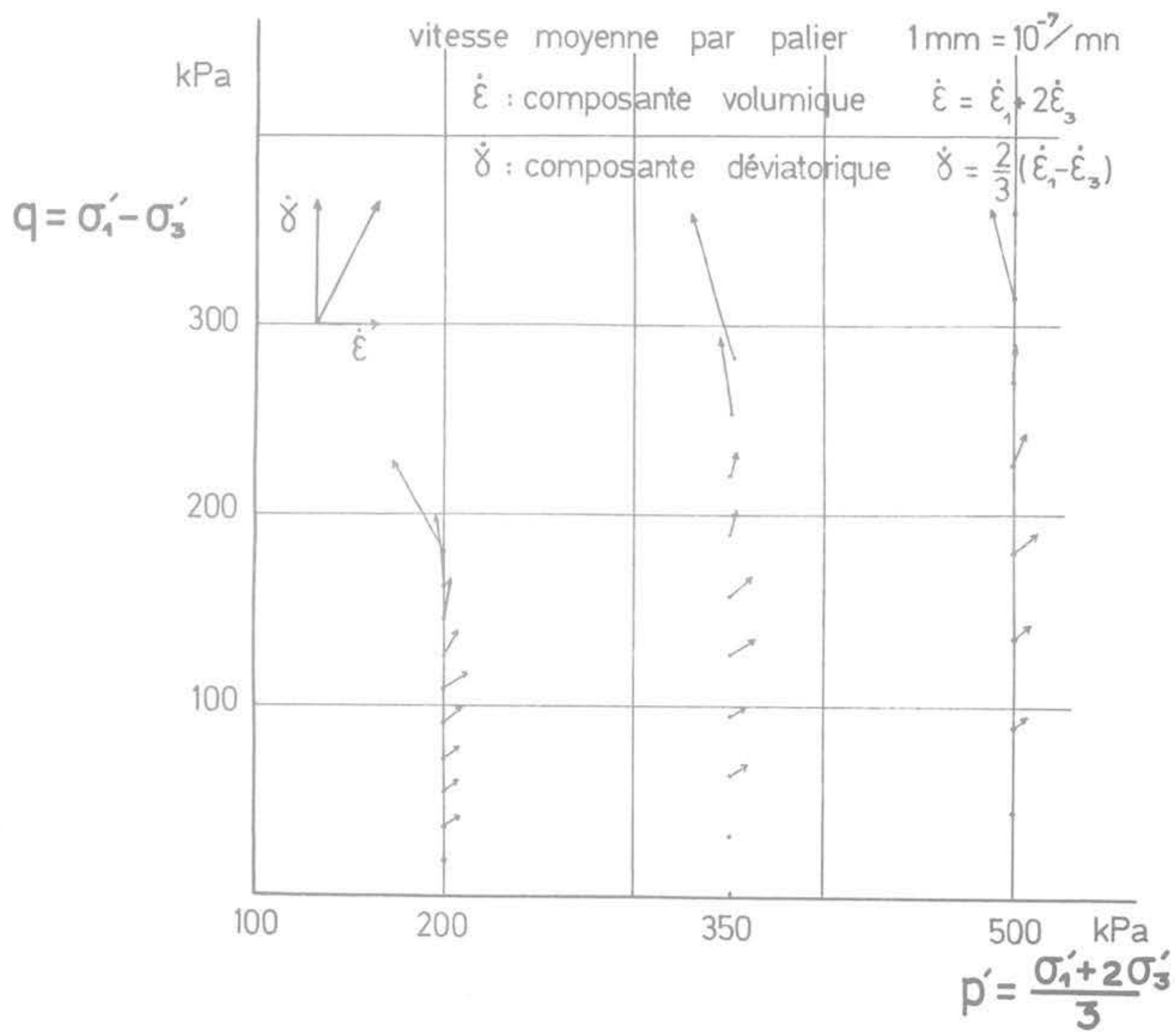

Fig. 6 Vecteurs vitesse de fluage moyenne associés aux différents paliers de charges appliqués à trois échantillons consoli. dés sous trois pressions isotropes $p^{\prime}$ différentes 
fonction des déformations déviatoriques relatives aux 4 essais de fluage au cisaillement sous charge constante et à l'essai de fluage sous chargement croissant par paliers. Les points représentent l'état de déformation des échantillons après 7 jours. Les chiffres sont égaux au rapport $\frac{q}{q_{\text {rupt }}}$ caractéristique des divers chargements ou paliers de chargement.

Ces résultats montrent que :

- Le taux de dilatance $\frac{d \theta}{d D}$ ne dépend pas significativement du temps. Les courbes sont des droites dans le cas des quatre chargements constants et une succession de segments correspondant aux différents paliers de chargement dans l'autre cas.

- La pente des droites et des segments diminue à mesure que le niveau de contrainte $\frac{q}{q_{\text {rupt }}}$ augmente. Sekiguchi (73) a présenté des résultats concordants pour une argile molle remaniée. II a observé toutefois une importante déformation déviatorique à volume quasi constant juste après le chargement, ce que la figure 5 ne montre pas.

- Le chargement par paliers provoque des déformations volumiques sensiblement plus importantes que les chargements constants, mais l'évolution indiquée dans les deux cas est la même.

- A l'approche de la rupture, le sens de variation de la déformation volumique s'inverse. L'argile des Flandres qui est constituée de multiples polyèdres imbriqués se disloque et augmente de volume. C'est ce que montre la figure 6 qui représente pour chacun des paliers de chargement des 3 essais le vecteur de vitesse moyenne de fluage de composantes

volumique : $\quad \dot{\varepsilon}=\frac{\Delta\left(\varepsilon_{1}+2 \varepsilon_{3}\right)}{\Delta t}$

déviatorique : $\dot{\gamma}=\frac{2}{3} \frac{\Delta\left(\varepsilon_{1}-\varepsilon_{3}\right)}{\Delta t} \quad \Delta t=7$ jours.

A la rupture, l'argile des Flandres n'atteint pas l'état critique au sens du modèle Cam Clay; le volume de l'argile augmente continuement.

Le champ de vitesse d'écoulement comme le laissait prévoir l'analyse de la figure 4 est paramétrable en fonction de $\frac{q}{p^{\prime}+a}$.

5 Mise en évidence de l'anisotropie, de la dilatance et des caractéristiques d'écoulement de l'argile de Cubzac-les-Ponts

\subsection{Chemins de contraintes et chemins de déformations}

Pour un matériau isotrope, non dilatant et linéaire, chemins de contraintes et chemins de déformations finales (après achèvement des déformations différées) coïncident. La figure 7 montre avec clarté que l'argile de Cubzac-les-Ponts ne possède pas tout ou partie des propriétés précédentes.

La consolidation pendant 36 jours sous une contrainte isotrope $\mathrm{p}^{\prime}=80 \mathrm{kPa}$ s'accompagne pour les 6 échantillons d'une déformation déviatorique négative comprise entre $-0,022$ et $-0,034$. En d'autres termes, les déformations radiales sont plus grandes que les déformations verticales. Cette anisotropie de comportement s'explique par les conditions de consolidation in situ sous des contraintes principales verticales et horizontales $\sigma_{v}^{\prime}$ et $\sigma_{h}^{\prime}$ liées par un coefficient de poussée des terres au repos $K_{0}=\frac{\sigma_{h}^{\prime}}{\sigma_{v}^{\prime}}=0,48$ très différent de celui de l'essai $(K=1)$. Ce comportement est propre aux argiles normalement consolidées ou faiblement surconsolidées.

Les échantillons 1,2 et 3 soumis à 4 paliers de chargement de cisaillement égaux accompagnés de 4 paliers de déchargement de la composante isotrope qui les mènent à la rupture, présentent tous trois des déformations volumiques non négligeables sous les deux premiers paliers. II faut y voir l'achèvement au-delà des 36 jours de consolidation des déformations volumiques différées provoquées par la contrainte isotrope $\mathrm{p}^{\prime}=80 \mathrm{kPa}$.

Contrairement à ce qui a été observé sur l'argile des Flandres, les déformations déviatoriques à la rupture et à la fin des paliers antérieurs ne sont pas égales.

Les échantillons 4,5 et 6 soumis à 4 paliers de chargement isotropes égaux accompagnés d'une augmentation de la composante déviatorique $\Delta q=\zeta \Delta p^{\prime}$ avec $\zeta=0,75 ; 0,3$ et 0 , subissent des déformations déviatoriques en rapport. Pour $\zeta=0$, le chargement isotrope de consolidation provoque des déformations déviatoriques qui vont en diminuant avec l'application des paliers successifs. Ceux-ci détruisent progressivement la structure initiale et l'anisotropie de comportement qui la caractérisait.

Sur les courbes de la figure 7 , les déformations qui se développent dans les 60 premières minutes sont omises. Les blancs correspondants sont négligeables par rapport aux segments correspondant à chaque palier: la déformation de l'argile est de nature essentiellement visqueuse.

\subsection{L'écoulement visco-plastique de l'argile de Cubzac-les-Ponts}

Les vitesses de déformation moyennes de l'argile durant les 60 premières minutes de chargement sont représentées sur la figure 8 par des vecteurs dont l'origine est située au point $\mathrm{p}^{\prime}, \mathrm{q}$ caractéristique du palier de chargement correspondant. La figure 9 représente de la même manière les vecteurs vitesse à la fin de chacun des paliers qui sont 100 fois plus petites, approximativement, que les précédentes.

L'orientation des vecteurs écoulement ne dépend pas significativement du temps. D'après la théorie de la visco-plasticité de Perzyna (63), la direction du vecteur vitesse d'écoulement est normale à la courbe d'état limite dynamique - équation (4) - et son intensité dépend de la différence réduite $\frac{\mathrm{Fd}-\mathrm{Fs}}{\mathrm{Fs}}$ entre la CELD et la CELS. Celle-ci diminue du fait de l'élargissement de la CELS par écrouissage. Ce schéma dans ses grandes lignes s'applique aux résultats des présents essais.

\section{Conclusion}

Les modèles de comportement en compression triaxiale (directions principales des contraintes et des déformations fixes) pour les sols visqueux sont relativement nombreux et ont pour point de départ les 


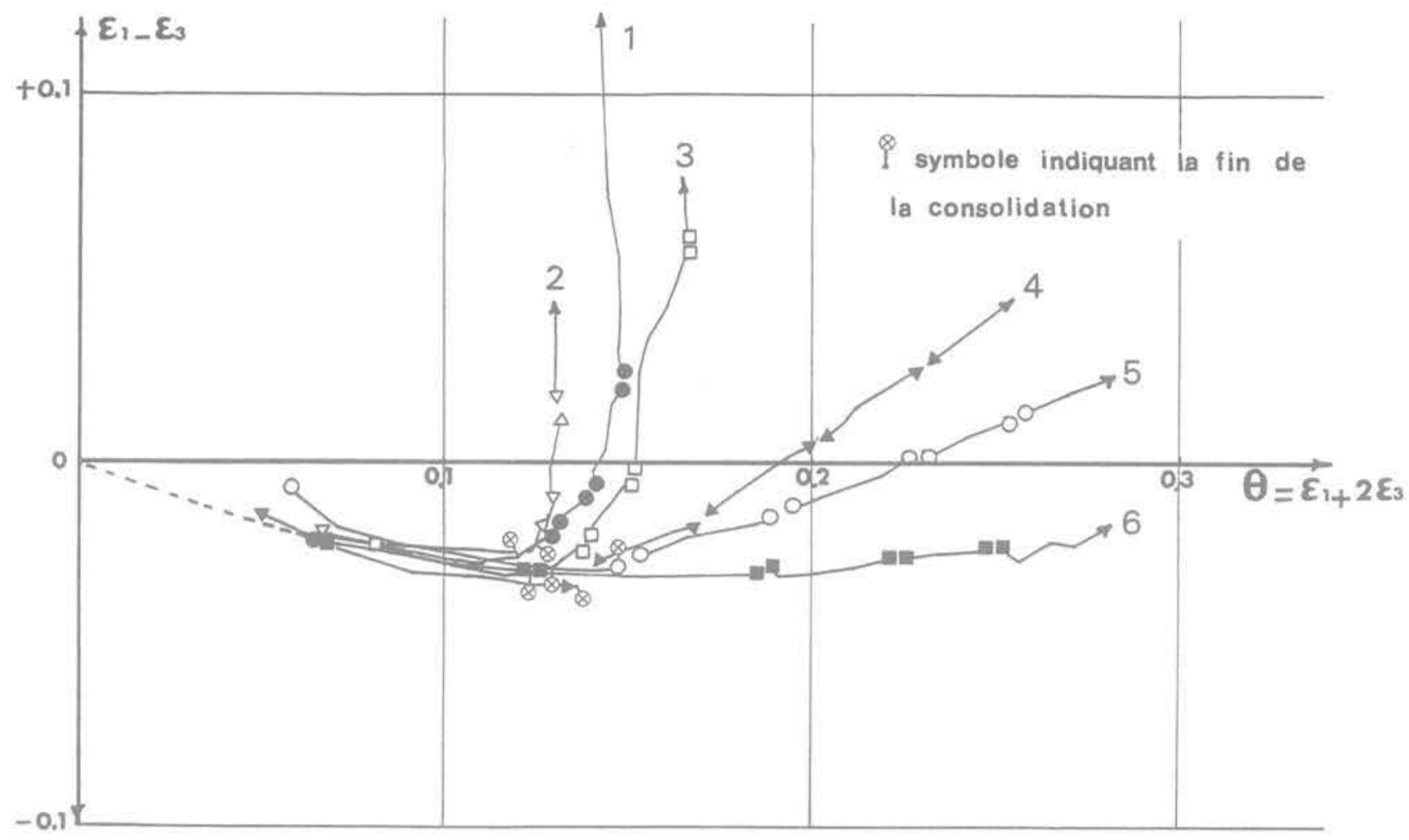

Fig. 7 Chemins de deformation suivis par six échantillons d'argile de Cubzac-les-Ponts sous six chemins de chargement différents

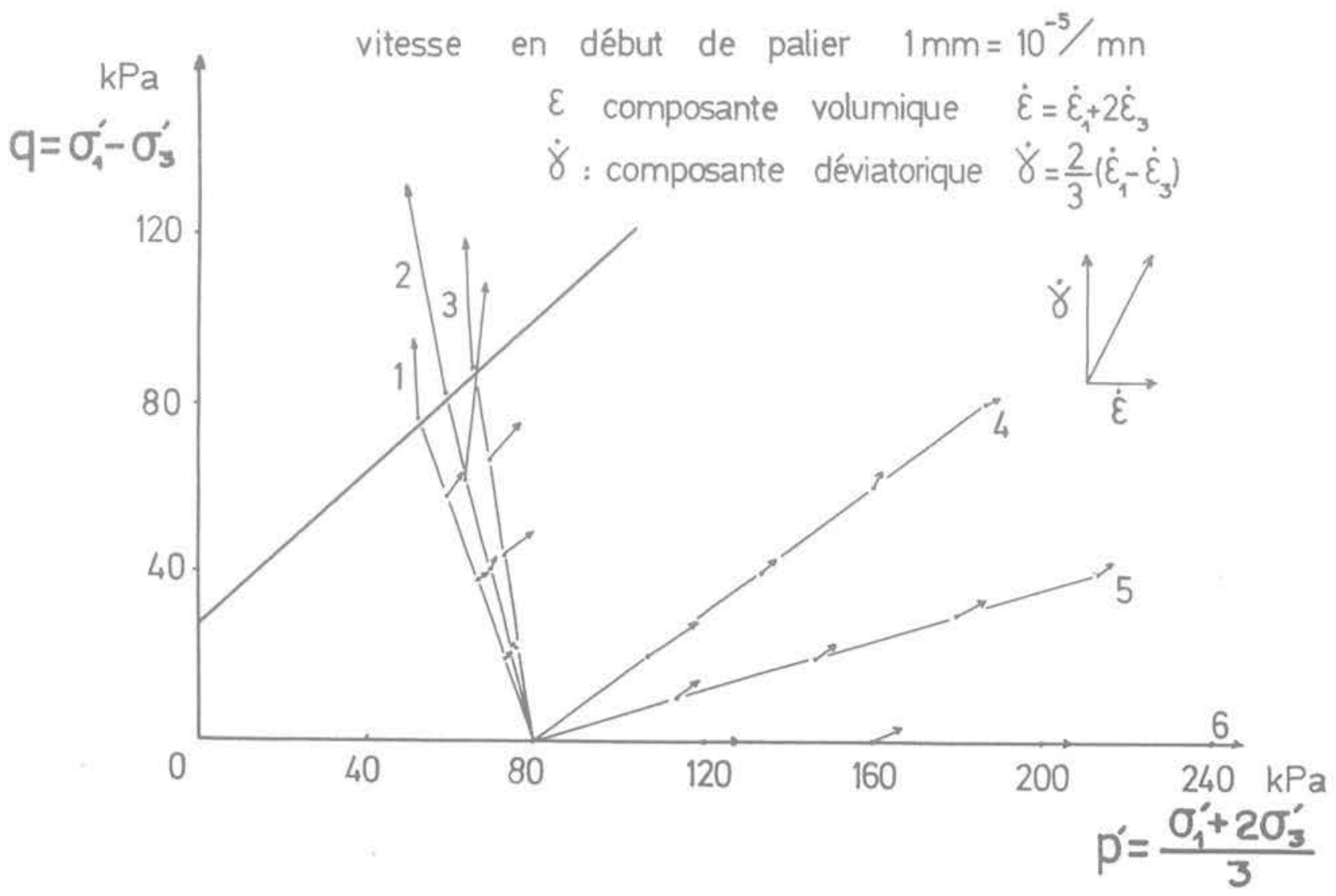

Fig. 8 Vitesses d'écoulement juste après les différents chargements 


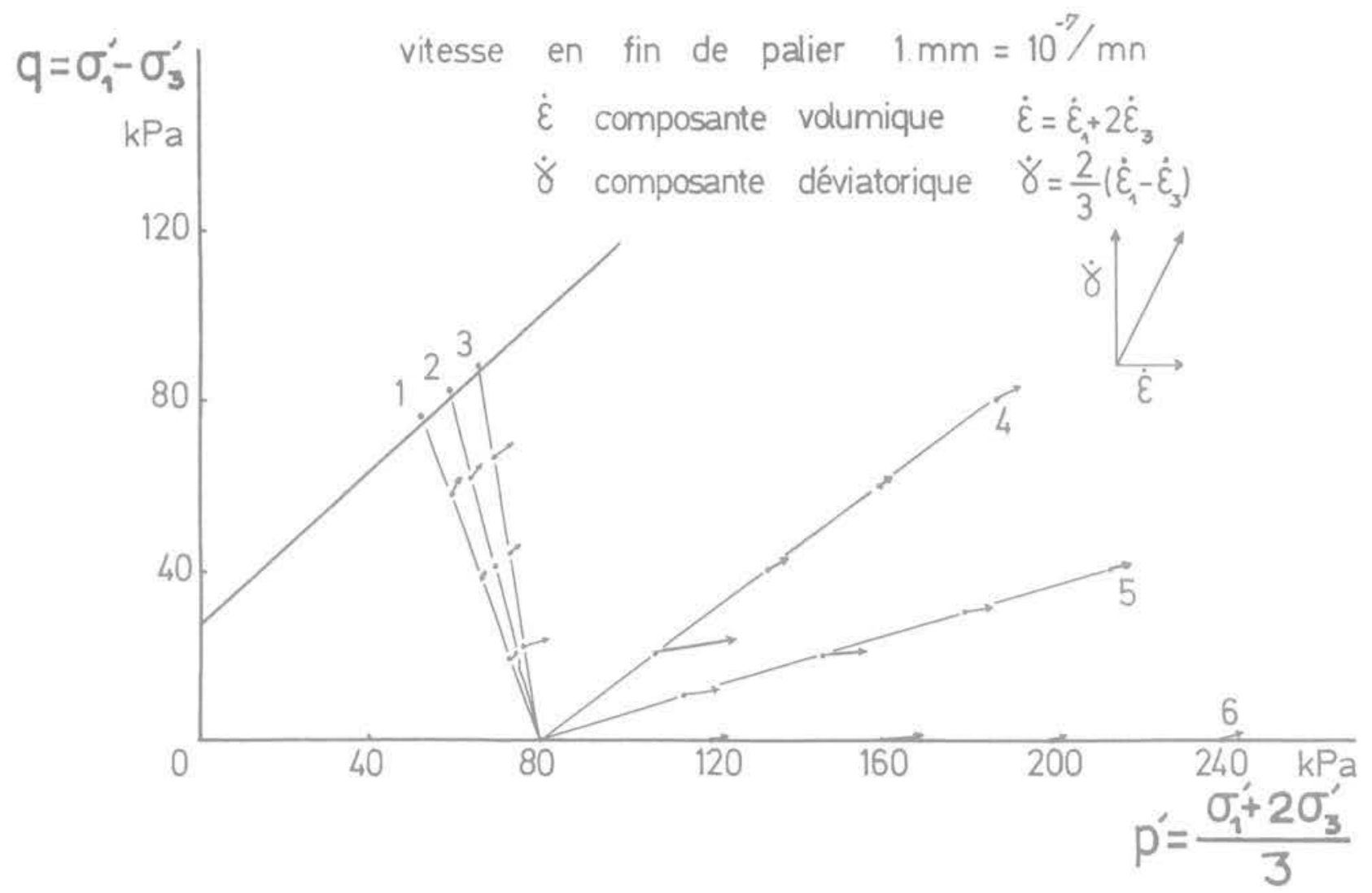

Fig. 9 Vitesses d'écoulement à la fin de chaque palier de chargement

théories de la visco-élasticité ou de l'élasto-viscoplasticité. Pour rester intelligible, chaque modèle doit se limiter à des types de sollicitations bien déterminés. C'est ainsi qu'un modèle visco-élastique basé sur le principe de superposition peut être envisagé pour la simulation du fluage au cisaillement d'une argile très compacte (l'argile des Flandres) dans les conditions particulières suivantes: consolidation isotrope puis augmentation instantanée ou par paliers des seules contraintes de cisaillement. II n'a pas été difficile de montrer, par des essais effectués sur une argile molle non remaniée, prélevée à Cubzac-les-Ponts, que l'application de ce modèle à d'autres types de chargements et à d'autres types d'argiles se heurtait à des difficultés dues en particulier à la dilatance, à l'anisotropie de comportement, à l'irréversibilité des øéformations visqueuses, etc.

\section{Remerciements}

Les auteurs expriment toute leur gratitude pour l'aide apportée par M. Stempfelet, technicien au LCPC, à la mise au point des bagues de mesure des déformations radicales et à la réalisation des essais de fluage sur l'argile de Cubzac-les-Ponts.

Ils adressent également leurs remerciements à M. Maebe, technicien au LRPC de Lille, et à M. Ghesquiers, technicien au LCPC, pour la bonne réalisation des essais de fluage sur l'argile des Flandres.

\section{Références bibliographiques}

Adachi T., Okano M. (1974), A constitutive equation for normally consolidated clay soils and foundations, vol. XIV, $n^{\circ} 4$, december, pp. 55-74.

Akai K, Adachi T., Fujimoto K. (1977), Constitutive equations of geomechanical materials based on elasto-viscoplasticity.

$9^{\circ}$ congrès int. MS et TF, Tokyo, Speciality session 9 , p. $1-10$.

Ansal M.A., Bazant Z.P., Krizek R.J. (1979), Viscoplasticity of normally consolidated clay.

Journal ASCE, GED, vol. 105 GT4, April, pp. 519-538.

Bishop A.W., Lovenbury H.T. (1969), Creep characteristics of two undisturbed clays.

$7^{\circ}$ congrès int. MS et TF, Mexico, vol. I, pp. 29-38.

Félix B. (1980), Étude théorique et expérimentale du fluage unidimensionnel des sols argileux.

Revue Française de Géotechnique $n^{\circ} 13$, novembre, pp. 31-43.

Félix B. (1981), Mesure de la déformation radiale des éprouvettes de sol par un système de bagues. Revue Française de Géotechnique, n 15, mai, pp. 53 57. 
Goldstein M., Ter Stepanian (1957), The long-term strength of clays and depth creep of siopes.

$7^{\circ}$ Congrès int. MS et TF, Londres, vol. II, pp. 311-314.

Meschyan S.R. (1967), Le fluage des sols argileux (en russe).

Édition de l'Académie des Sciences de la RSS d'Arménie, p. 318.

Meschyan S.R., Badalyan R.G. (1976), Regularity of creep of clays and deformation of slopes.

$6^{\circ}$ conf. Européen. MS et TF, Vienne, vol. 1-1, pp. 71-74.

Murayama S., Shibata T. (1961), Rheological properties of clays.

$5^{\circ}$ congrès int. MS et TF, Paris, vol. I, pp. 269-273.

Perzyna P. (1969), The constitutive equations for work-hardening and rate sensitive plastic material.

Proceedings Vibrational Problems, Varsovie, vol. 14, n०3, pp. 281-290.

Roscoe K.H., Schofield A.N., Wroth C.P. (1958), On the yieiding of soils. Geotechnique, vol. VIII, n 1, pp. 2253.

Robinet J.-C. (1978), Prédiction du comportement d'une argile sur chemins triaxiaux.

Journée de Rhéologie, ENTPE, Vaulx-en-Velin, pp. 55 98.
Schultze E. (1971), Essais de fluage sur des sols normalement compactés. Bull. de Liaison des LPC Spécial journées nationales :

Le comportement des sols avant la rupture, Paris, Mai, pp. 20-29.

Sekiguchi H. (1973), Flow characteristics of clays Soils and Foundations, vol. XIII, $n^{\circ} 1$, March, pp. 45-60.

Sekiguchi H., Ohta H. (1977), Induced anisotropy and time dependancy in clays.

$9^{\circ}$ congrès int. MS et TF, Tokyo, Specialty session 9 , pp. 229-238.

Shahanguian S. (1981), Détermination expérimentale des courbes d'état limite de l'argile organique de Cubzac-les-Ponts.

Rapport de Recherche LPC $n^{\circ} 106$, novembre, p. 79.

Shibata T., Karube D. (1969), Creep rate and creep strength of clays.

$7^{\circ}$ congrès int. MS et TF, Mexico, vol. I, pp. 351-360.

Tavenas F., Leroueil S. (1977), Effects of stresses and time on yielding of clays.

$9^{\circ}$ congrès int. MS et TF, Tokyo, vol. I, pp. 319-326.

Valanis K.C. (1971), A theory of viscoplasticity without a yield surface. Archive de mécanique (Archiwum Mechaniki Stosowanej), $n^{\circ} 23$, pp. 517-555.

Vyalov S.S., Skibitskij A.M. (1957), Rheological processes in frozen soils and dense clays.

$4^{\circ}$ congrès int. MS et TF, Londres, vol. I, pp. 120-124. 